ARTICLE

https://doi.org/10.1038/s41467-019-10693-0

\title{
Multiferroicity in atomic van der Waals heterostructures
}

\author{
Cheng Gong ${ }^{1}$, Eun Mi Kim², Yuan Wang ${ }^{1,3}$, Geunsik Lee (iD ${ }^{2}$ \& Xiang Zhang (1) ${ }^{1,3}$
}

Materials that are simultaneously ferromagnetic and ferroelectric - multiferroics - promise the control of disparate ferroic orders, leading to technological advances in microwave magnetoelectric applications and next generation of spintronics. Single-phase multiferroics are challenged by the opposite $d$-orbital occupations imposed by the two ferroics, and heterogeneous nanocomposite multiferroics demand ingredients' structural compatibility with the resultant multiferroicity exclusively at inter-materials boundaries. Here we propose the two-dimensional heterostructure multiferroics by stacking up atomic layers of ferromagnetic $\mathrm{Cr}_{2} \mathrm{Ge}_{2} \mathrm{Te}_{6}$ and ferroelectric $\mathrm{In}_{2} \mathrm{Se}_{3}$, thereby leading to all-atomic multiferroicity. Through first-principles density functional theory calculations, we find as $\ln _{2} \mathrm{Se}_{3}$ reverses its polarization, the magnetism of $\mathrm{Cr}_{2} \mathrm{Ge}_{2} \mathrm{Te}_{6}$ is switched, and correspondingly $\mathrm{In}_{2} \mathrm{Se}_{3}$ becomes a switchable magnetic semiconductor due to proximity effect. This unprecedented multiferroic duality (i.e., switchable ferromagnet and switchable magnetic semiconductor) enables both layers for logic applications. Van der Waals heterostructure multiferroics open the door for exploring the low-dimensional magnetoelectric physics and spintronic applications based on artificial superlattices.

\footnotetext{
${ }^{1}$ Nano-scale Science and Engineering Center (NSEC), 3112 Etcheverry Hall, University of California, Berkeley, CA 94720, USA. ${ }^{2}$ Department of Chemistry, Ulsan National Institute of Science and Technology, Ulsan 44919, Korea. ${ }^{3}$ Materials Sciences Division, Lawrence Berkeley National Laboratory, 1 Cyclotron Road, Berkeley, CA 94720, USA. Correspondence and requests for materials should be addressed to G.L. (email: gslee@unist.ac.kr) or to X.Z. (email: xiang@berkeley.edu)
} 
$\mathrm{M}$ ultiferroics, a class of functional materials that simultaneously possess more than one ferroic orders such as ferromagnetism and ferroelectricity, hold great promise in magnetoelectric applications due to the inherent coupling between ferroic orders ${ }^{1-6}$, leading to technological advances in next generation of spintronics and microwave magnetoelectric devices. However, single-phase multiferroics are challenged by the different ferroics' contradictory preference on the d-orbital occupation of metal ions: ferroelectricity arising from off-center cations requires empty $d$-orbitals, whereas ferromagnetism usually results from partially filled $d$-orbitals ${ }^{7}$. Conventional perovskite multiferroics (chemical formula: $\mathrm{ABO}_{3}$ ) have lonepair-active A-sites which move to off-centers of centrosymmetric crystals for electric polarization, and B-sites with unpaired electrons for magnetic order. Because the ferroelectric and magnetic order in these materials are associated with different ions, the coupling between the ferroic orders are usually weak.

Heterogeneous multiferroics, synthesized composites of two mixed phases ${ }^{8}$, have the coupling between ferroelectric and magnetic order exclusively at inter-materials boundaries, with magnetoelectric effects occasionally established via interfacial magnetoelastic effect. As an example, magnetic nanopillars could be embedded in ferroelectric matrix. However, these heterogeneous multiferroics stringently demand the constituent materials on their structural similarity, lattice match and chemical compatibility, and have weak magnetoelectric effects limited by the interface/bulk ratios.

Van der Waals (vdW) crystals emerged as ideal material systems with unprecedented freedom for heterostructure construction ${ }^{9}$. Recent experimental advance discovered ferromagnetism ${ }^{10-12}$ and ferroelectricity ${ }^{13}$ in different two-dimensional vdW crystals separately. It remains a paramount challenge to realize multiple ferroic orders in a single-phase $2 \mathrm{D}$ material simultaneously ${ }^{14-17}$, as each order encounters its own challenge (e.g., ferromagnetism in 2D systems suffers from enhanced thermal fluctuations, whereas ferroelectricity the depolarization field). Constructing heterostructures of $2 \mathrm{D}$ magnets and $2 \mathrm{D}$ ferroelectrics potentially provides a generally applicable route to create $2 \mathrm{D}$ multiferroics. However, the fundamental question remains regarding whether the interlayer magnetoelectric coupling can be established, given the presence of the interlayer $\mathrm{vdW}$ spacing. If realized, layered heterostructure multiferroics would provide completely new platforms with all atoms participating in the inter-ferroics coupling, and largely reshape the landscape of multiferroics based on vdW superlattices.

Through first-principles density functional theory (DFT) calculations based on a bilayer heterostructure of ferromagnetic $\mathrm{Cr}_{2} \mathrm{Ge}_{2} \mathrm{Te}_{6}$ and ferroelectric $\mathrm{In}_{2} \mathrm{Se}_{3}$ monolayers ${ }^{18-21}$, we discovered a strong interlayer magnetoelectric effect: the reversal electric polarizations in $\operatorname{In}_{2} \mathrm{Se}_{3}$ switches the magnetocrystalline anisotropy of $\mathrm{Cr}_{2} \mathrm{Ge}_{2} \mathrm{Te}_{6}$ between out-of-plane and in-plane orientations. For a $2 \mathrm{D}$ ferromagnet, such a change in magnetic anisotropy corresponds to a switching on/off of the ferromagnetic order at finite temperatures, for easy-axis anisotropy opens spin wave excitation gap and thus suppresses the thermal fluctuations, but easy-plane anisotropy does not ${ }^{10,22,23}$. The switching of ferromagnetic order by electric polarization promises a novel design of magnetic memory. Detailed analysis unraveled the interfacial hybridization as the cause of interlayer magnetoelectric coupling. Furthermore, $\mathrm{In}_{2} \mathrm{Se}_{3}$ becomes magnetized due to the proximity to $\mathrm{Cr}_{2} \mathrm{Ge}_{2} \mathrm{Te}_{6}$, making $\mathrm{In}_{2} \mathrm{Se}_{3}$ a single-phase multiferroics (i.e., ferromagnetic and ferroelectric orders coexist in $\mathrm{In}_{2} \mathrm{Se}_{3}$ ), although apparently the magnetization of $\mathrm{In}_{2} \mathrm{Se}_{3}$ requires the presence of the adjacent $\mathrm{Cr}_{2} \mathrm{Ge}_{2} \mathrm{Te}_{6}$. Such multiferroicity duality-that is, the interlayer multiferroicity and the $\mathrm{In}_{2} \mathrm{Se}_{3}$ intralayer multiferroicity - provides unique solid-state system in which ferroelectric and ferromagnetic orders interplay inherently. This unusual multiferroicity duality in vdW heterostructures may open avenues for developing new concepts of magnetoelectric devices: using single knob (the orientation of electric polarization in $\mathrm{In}_{2} \mathrm{Se}_{3}$ ) to control the magnetic order in both $\mathrm{In}_{2} \mathrm{Se}_{3}$ and $\mathrm{Cr}_{2} \mathrm{Ge}_{2} \mathrm{Te}_{6}$. We envision the multiferroicity duality potentially enriches the freedom of layer-resolved data storage and that of information processing due to the diverse magnetoelectric and magneto-optic properties of constituent layers.

\section{Results}

Material model and computational details. In this work, the lattice constant of $\mathrm{Cr}_{2} \mathrm{Ge}_{2} \mathrm{Te}_{6}$ adopted the experimental value $6.83 \AA$ and was fixed in heterostructures for the sake of minimizing artifact effects, considering the magnetic properties of $2 \mathrm{D}$ $\mathrm{Cr}_{2} \mathrm{Ge}_{2} \mathrm{Te}_{6}$ are sensitive to structure parameters. It has been reported that a monolayer $\mathrm{In}_{2} \mathrm{Se}_{3}$ of either zincblende or wurtzite stacking is unstable with a tendency of the lateral displacement of the top Se layer, leading to the energetically degenerate ferroelectric monolayer ${ }^{18}$. Although the one relaxed from the zincblende stacking is chosen in this study, it will be also applicable to the other derived from the wurtzite, because the main mechanism to be shown is determined by the interfacial monolayers and thus independent of the detailed stacking type of the multilayer $\operatorname{In}_{2} \mathrm{Se}_{3}$. The optimized lattice constant of $1 \times 1-\mathrm{In}_{2} \mathrm{Se}_{3}(4.106 \AA)$, is strained by $-4.0 \%$ to match $\operatorname{In}_{2} \mathrm{Se}_{3}-\sqrt{3} \times \sqrt{3}$ to $\mathrm{Cr}_{2} \mathrm{Ge}_{2} \mathrm{Te}_{6}-1 \times 1$ as shown by Fig. 1a. In heterostructure, the relative spacing and registry between $\mathrm{Cr}_{2} \mathrm{Ge}_{2} \mathrm{Te}_{6}$ and $\mathrm{In}_{2} \mathrm{Se}_{3}$ are adjusted to find the energy minimum configuration. The reversal of the electric polarization of the isolated $\operatorname{In}_{2} \mathrm{Se}_{3}$ monolayer can be achieved via lateral displacement of the middle most Se layer, with an energy barrier as small as $0.04 \mathrm{eV}$ per formula unit estimated by nudged elastic band calculation ${ }^{24}$. In heterostructure, due to the large vdW spacing, the presence of $\mathrm{Cr}_{2} \mathrm{Ge}_{2} \mathrm{Te}_{6}$ does not noticeably affect the energy barrier of the electric-polarization reversal process of $\mathrm{In}_{2} \mathrm{Se}_{3}$. The total energy of the heterostructure is lowest (highest) where the interfacial Te atoms sit at the hollow (top) site
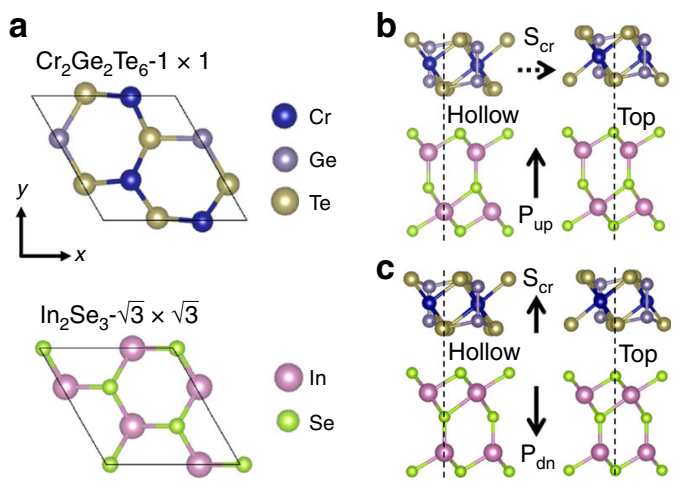

Fig. 1 Van der Waals $\mathrm{Cr}_{2} \mathrm{Ge}_{2} \mathrm{Te}_{6} / \mathrm{In}_{2} \mathrm{Se}_{3}$ heterostructure and magnetoelectric coupling. a Lateral unit cell size is chosen to be equal to that of ferromagnetic $\mathrm{Cr}_{2} \mathrm{Ge}_{2} \mathrm{Te}_{6}$ experimental lattice constant and commensurate to ferroelectric $\ln _{2} \mathrm{Se}_{3}-\sqrt{3} \times \sqrt{3}$ with $-4 \%$ strain. The interfacial two atomic layers (one atomic layer from each side of the interface) are shown in the top views of $\mathrm{Cr}_{2} \mathrm{Ge}_{2} \mathrm{Te}_{6}-1 \times 1$ (top) and $\operatorname{In}_{2} \mathrm{Se}_{3}$ $\sqrt{3} \times \sqrt{3}$ (bottom). b, c Heterostructure side views with the $\ln _{2} \mathrm{Se}_{3}$ ferroelectric dipole moment directed upward and downward ( $\mathbf{P}_{\mathbf{u p}}$ and $\mathbf{P}_{\mathbf{d n}}$ ), respectively, and the induced easy-plane and easy-axis $\mathrm{Cr}$ spins $\left(\mathbf{S}_{\mathbf{C r}}\right)$. The solid (dashed) arrow of $\mathbf{S}_{\mathbf{c r}}$ indicates the allowed (prohibited) finite temperature long-range ferromagnetic ordering in 2D systems by the Mermin-Wagner theorem. For each case, the most stable (hollow) and unstable (top) stacking configurations are given 
of $\mathrm{In}_{2} \mathrm{Se}_{3}$, with their relative energy difference amounts to 0.31 and $0.35 \mathrm{eV} /$ u.c. for upward (Fig. 1b) and downward (Fig. 1c) polarizations, respectively. The equilibrium interlayer distance between $\mathrm{Cr}_{2} \mathrm{Ge}_{2} \mathrm{Te}_{6}$ and $\mathrm{In}_{2} \mathrm{Se}_{3}$ at the hollow configuration is 3.20 and $3.14 \AA$ for up and down polarizations of $\operatorname{In}_{2} \mathrm{Se}_{3}$, respectively. The total energy of the down polarization (Fig. 1c) is lower by $0.07 \mathrm{eV} /$ u.c. than that of the up polarization (Fig. 1b), due to the stronger interfacial coupling between downpolarized $\mathrm{In}_{2} \mathrm{Se}_{3}$ and $\mathrm{Cr}_{2} \mathrm{Ge}_{2} \mathrm{Te}_{6}$.

In order to reproduce the experimental magnetic properties of bulk $\mathrm{Cr}_{2} \mathrm{Ge}_{2} \mathrm{Te}_{6}$, we used small onsite Hubbard $U$ value $0.5 \mathrm{eV}$ and Hund's coupling $J$ value $0.0 \mathrm{eV}$ for $\mathrm{Cr} d$ orbital in DFT calculations (see ref. ${ }^{10}$ for the choice of $U=0.5 \mathrm{eV}, J=0.0 \mathrm{eV}$ ). This small onsite Columbic interaction is consistent with the fact that $\mathrm{Cr}_{2} \mathrm{Ge}_{2} \mathrm{Te}_{6}$ is a small band gap material with less localization than Cr-oxides. The ferromagnetic ground state is confirmed with the $\mathrm{Cr}$ spin magnetic moment $\sim 3.0 \mu_{\mathrm{B}}$. With the spin-orbit coupling (SOC) included, the magnetocrystalline anisotropy energy (MAE) is calculated and defined as $E_{[100]}-E_{[001]}$, where the former and latter correspond to the total energy with the $\mathrm{Cr}$ spins directed in-plane and out-of-plane, respectively. Due to the threefold rational symmetry of $\mathrm{Cr}_{2} \mathrm{Te}_{2} \mathrm{Te}_{6}$, there is not much magnetic anisotropy within the basal plane. We checked the convergence of MAE carefully, where a large value of K-mesh $(12 \times 12 \times 1)$ was enough to ensure the error $<10 \mu \mathrm{eV} / \mathrm{Cr}$. For the isolated monolayer $\mathrm{Cr}_{2} \mathrm{Ge}_{2} \mathrm{Te}_{6}$, our calculated MAE is $-70 \mu \mathrm{eV} / \mathrm{Cr}$, favoring the in-plane direction. In the heterostructures with up- and downpolarized $\mathrm{In}_{2} \mathrm{Se}_{3}$, the calculated $\mathrm{Cr}$ MAE is -95 and $75 \mu \mathrm{eV}$, respectively, whose energetically favorable spin orientations are indicated by $\mathbf{S}_{\mathrm{Cr}}$ in Fig. 1b, c. By modulating the polarization of the adjacent ferroelectric layer, the switching of the magnetization orientation is realized. This has significant application implications: For a 2D magnetic system with easy-plane anisotropy (X-Y model), the finite temperature ferromagnetic order is inhibited, whereas for easy-axis anisotropy (Ising model), the magnetic order can be sustained at finite temperatures. Therefore, in such a heterostructure, multiferroic effect offers a potential route to switch the ferromagnetism for logic devices.

Mechanism for interfacial multiferroicity. The mechanism for electric-polarization dependent MAE is discussed in details. The calculated Cr orbital moment is small $\left(<\mathrm{L}_{\mathrm{x}}\right\rangle=0.04 \mu_{\mathrm{B}},\left\langle\mathrm{L}_{\mathrm{z}}\right\rangle=$ $0.01 \mu_{\mathrm{B}}$ for in-plane, out-of-plane spin directions), which is less likely the origin of MAE. The plausible mechanism is related to the detailed feature of spin-resolved orbital-decomposed band structure ${ }^{25}$. Starting from the collinear spin band structures, we analyzed the energy correction by the perturbation theory about $\lambda \mathbf{L} \cdot \mathbf{S}$ where $\lambda$ is the radial part of $\mathrm{Cr}$ SOC. The orbital moment quenched by the crystal field results in the vanishing first order correction. Assuming the negligible change of the electron correlation energy between [100] and [001] spin directions, one can write the second order contribution to MAE as follows ${ }^{25}$ :

$$
\begin{aligned}
\mathrm{MAE}= & \lambda^{2} \sum_{v, c, \sigma}\left(\left|\left\langle v, \sigma\left|L_{z}\right| c, \sigma\right\rangle\right|^{2}-\left|\left\langle v, \sigma\left|L_{x}\right| c, \sigma\right\rangle\right|^{2}\right) /\left(\epsilon_{c, \sigma}-\epsilon_{v, \sigma}\right) \\
& +\lambda^{2} \sum_{v, c, \sigma \neq \sigma^{\prime}}\left(\left|\left\langle v, \sigma\left|L_{x}\right| c, \sigma^{\prime}\right\rangle\right|^{2}-\left|\left\langle v, \sigma\left|L_{z}\right| c, \sigma^{\prime}\right\rangle\right|^{2}\right) /\left(\epsilon_{c, \sigma^{\prime}}-\epsilon_{v, \sigma}\right),
\end{aligned}
$$

Here the first and second summations correspond to the spinconserving $\left|\Delta s_{\mathrm{z}}\right|=0$ and spin-flipping $\left|\Delta s_{\mathrm{z}}\right|=1$ transitions, and $|v, \sigma\rangle$ and $|c, \sigma\rangle$ are valence and conduction band states with spin $\sigma$, respectively, whose energy eigenvalues are $\epsilon_{c, \sigma}$ and $\epsilon_{v, \sigma}$. The angular momentum matrix elements of $\mathrm{Lz}$ and $\mathrm{Lx}$ correspond to transitions with $\left|\Delta m_{\mathrm{z}}\right|=0$ and $\left|\Delta m_{\mathrm{z}}\right|=1$, respectively, for $\mathrm{Cr} d$ - orbitals. Therefore, for spin-conserving transition, SOC elements between occupied and unoccupied states with the same (different) magnetic quantum number through the $L_{z}\left(L_{x}\right)$ operator contributes to positive (negative) MAE. For spin-flipping transition, the contribution to MAE is reversed ${ }^{26}$.

Figure $2 \mathrm{a}, \mathrm{b}$ shows the spin-resolved orbital-decomposed band structure of heterostructures for up- and downpolarized $\operatorname{In}_{2} \mathrm{Se}_{3}$, respectively, where the contribution of $\mathrm{Cr} d$-orbitals is indicated by the circles for $\left|m_{z}\right|=0\left(z^{2}\right), 1(x z$ and $y z)$, or $2\left(x^{2}-y^{2}\right.$ and $\left.x y\right)$. As shown by the arrows in Fig. $2 \mathrm{a}$ for the heterostructure with up-polarized $\mathrm{In}_{2} \mathrm{Se}_{3}$, our calculated negative MAE mainly originates from the spin-conserving transition from $\left|m_{\mathrm{z}}\right|=1$ to $\left|m_{\mathrm{z}}\right|=0$ or 2, i.e., $\left|\Delta s_{\mathrm{z}}\right|=0$ and $\left|\Delta m_{\mathrm{z}}\right|=1$ related to the second term of the first sum in Eq. (1). The mechanism is further confirmed by our results that the Cr MAE changes from about -100 to $200 \mu \mathrm{eV}$ by intentionally increasing the $U$ value from 0.5 to $2.0 \mathrm{eV}$ : the increased $U$ lowers the energy level of the majority spin in valence bands of $\left|m_{\mathrm{z}}\right|=0$ or 2 (Supplementary Fig. 3), and thus the transition energy gap of $\left|\Delta s_{\mathrm{z}}\right|=0$ and $\left|\Delta m_{\mathrm{z}}\right|=1$ is increased and the associated contribution is weakened, leading to the positive MAE.

However, for the heterostructure with downpolarized $\mathrm{In}_{2} \mathrm{Se}_{3}$, as shown in Fig. 2b, the conduction band minimum of Cr $d\left|m_{\mathrm{z}}\right|=$ 1 shows a large gap $(\sim 0.2 \mathrm{eV})$ near $0.5 \mathrm{eV}$ above Fermi level, which is caused by hybridization with the $\mathrm{In}_{2} \mathrm{Se}_{3}$ conduction band minimum. Such hybridization results in a significant depletion of $\left|m_{\mathrm{z}}\right|=1$ majority spin DOS (Supplementary Fig. 2a). Hence, the negative contribution to MAE found for the case of up-polarized $\mathrm{In}_{2} \mathrm{Se}_{3}$ is suppressed. Meanwhile the minority spin DOS remains almost unchanged for $\left|m_{\mathrm{z}}\right|=1$ (Supplementary Fig. 2a), leading to positive MAE via $\left|\Delta s_{\mathrm{z}}\right|=1$ and $\left|\Delta m_{\mathrm{z}}\right|=1$, as illustrated by the arrows in Fig. 2b. Considering the interfacial hybridization depends on the band alignment of $\mathrm{In}_{2} \mathrm{Se}_{3}$ and $\mathrm{Cr}_{2} \mathrm{Ge}_{2} \mathrm{Te}_{6}$, we employed the Heyd-Scuseria-Ernzerhof exchange-correlation functional (HSE06) to recalculate the band properties of the heterostructures. As expected, the calculated band gaps widen compared with the GGA-PBE results, but the key features while $\mathrm{In}_{2} \mathrm{Se}_{3}$ reverses its electric orientation from up to down keep the same: the conduction band of $\operatorname{~}_{2} \mathrm{Se}_{3}$ moves down to hybridize with the conduction band of $\mathrm{Cr}_{2} \mathrm{Ge}_{2} \mathrm{Te}_{6}$, as clearly seen in Supplementary Fig. 4.

The sign change of $\mathrm{Cr}_{2} \mathrm{Ge}_{2} \mathrm{Te}_{6}$ 's MAE upon the electricpolarization reversal of $\mathrm{In}_{2} \mathrm{Se}_{3}$ from up to down arises from the increased coupling, which causes the overall shift down of $\mathrm{In}_{2} \mathrm{Se}_{3}$ bands and its enhanced hybridization with $\mathrm{Cr}_{2} \mathrm{Ge}_{2} \mathrm{Te}_{6}$. This suggests that the positive MAE for the down polarization would be enhanced by a reduced vdW spacing. To confirm this scenario, we did interlayer spacing dependent MAE calculations for hollow configuration as shown in Fig. 3. As the interlayer distance decreases, MAE increases gradually with a slight fluctuation. The fluctuation originates from the detailed variation of energy levels in the spin-polarized band structures. From the same calculation conducted for the top configuration, it exhibits a stronger fluctuation with the interlayer spacing, due to the larger degree of interfacial orbital overlap in top configuration. The same trend of the two curves in Fig. 3 confirms that the increased interlayer hybridization tends to switch the magnetocrystalline anisotropy from easy-plane to easy-axis. Detailed spin-resolved orbitaldecomposed analysis in Supplementary Fig. 5 shows that the decreased spin-flipping energy gap near $\mathrm{K}$ with decreased interlayer distance contributes to the positive MAE.

Magnetized $\mathrm{In}_{2} \mathrm{Se}_{3}$ in proximity to $\mathrm{Cr}_{2} \mathrm{Ge}_{2} \mathrm{Te}_{6}$. Remarkably, the exchange splitting in $\mathrm{Cr} d$-band magnetizes $\mathrm{In}_{2} \mathrm{Se}_{3}$ by the proximity effect. As shown in Fig. 2, the highest valence band has a 
a
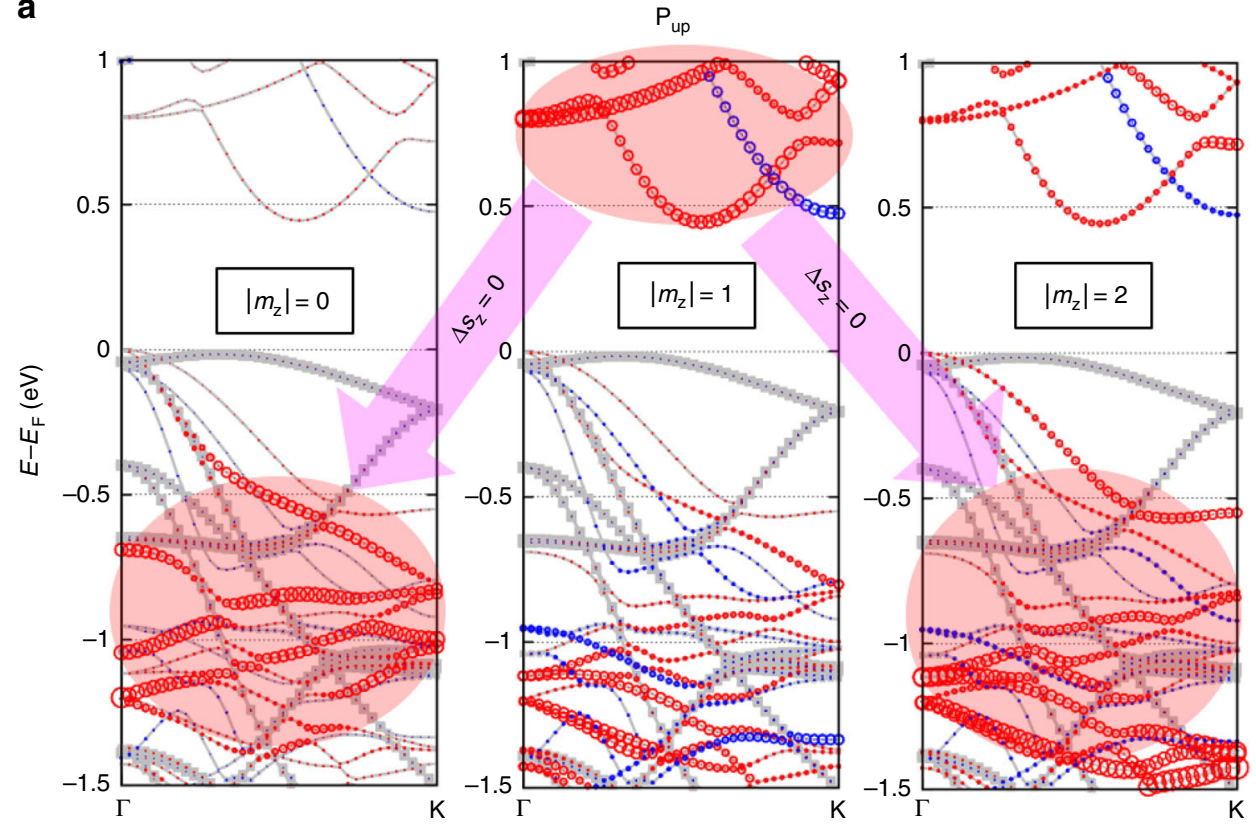

b


Fig. 2 Spin-polarized and $\mathrm{Cr}$ d orbital-decomposed band structures of $\mathrm{Cr}_{2} \mathrm{Ge}_{2} \mathrm{Te}_{6} / \mathrm{In}_{2} \mathrm{Se}_{3}$ heterostructures. $\mathbf{a}$, $\mathbf{b}$ The calculated band structures for the heterostructures with $\mathbf{P}_{\mathbf{u p}}$ and $\mathbf{P}_{\mathbf{d n}} \ln _{2} \mathrm{Se}_{3}$, respectively. For each electric polarization, the heterostructure (in terms of contact registry and interlayer distance) adopted the configuration of global energy minimum (see Supplementary Fig. 1). The majority and minority spin states are indicated by the red and blue circles, respectively, whose size denotes the contribution of $\mathrm{Cr} d$ orbitals with certain azimuthal angular momentum $\left|m_{z}\right| . \ln { }_{2} \mathrm{Se}_{3} \operatorname{states}$ are shown by the gray dots, and the band exhibits the overall shift down by $\sim 1 \mathrm{eV}$ while changing $\ln _{2} \mathrm{Se}_{3}$ from $\mathbf{P}_{\mathbf{u p}}$ and $\mathbf{P}_{\mathbf{d n}}$, which indicates a strong $\ln { }_{2} S e_{3}$-polarization dependent interfacial coupling. The pink arrows indicate the SOC elements between empty and filled states, indicated by the red circle for the majority spin or blue for minority, causing the negative or positive value of MAE (see Eq. (1) and text) for (a) or (b), respectively, thus the easy-plane or easy-axis $\mathbf{S}_{\mathbf{C r}}$

significant exchange splitting, where the majority-spin band is closer to the Fermi level near $\Gamma$. Those states are mainly caused by the surface $\mathrm{Te}$ atoms in $\mathrm{Cr}_{2} \mathrm{Ge}_{2} \mathrm{Te}_{6}$, which means the interfacial $\mathrm{Te}$ atoms have the electron spin antiparallel to that of $\mathrm{Cr} d$ electrons. Our calculated spin moment per Te atom is $-0.11 \mu_{\mathrm{B}}$ for $\mathrm{In}_{2} \mathrm{Se}_{3}$ of either electric polarizations. Also, the surface In and Se atoms has non-zero spin moments parallel to Te spins induced by the proximity. The spin-resolved DOS of interfacial In and Se, shown in Fig. 4a, confirmed the magnetized $\mathrm{In}_{2} \mathrm{Se}_{3}$. It is practically important to note the calculated magnetized $\mathrm{In}_{2} \mathrm{Se}_{3}$ here is a ground-state property. At finite temperatures, easy-plane magnetization of $2 \mathrm{D} \mathrm{In}_{2} \mathrm{Se}_{3}$ is susceptible to thermal fluctuations and long-range order does not exist, but easy-axis magnetization of $2 \mathrm{D} \mathrm{In}_{2} \mathrm{Se}_{3}$ could sustain the spin polarization at certain finite temperatures. Hence, a switching of $2 \mathrm{D}$ magnetic ferroelectric $\mathrm{In}_{2} \mathrm{Se}_{3}$ could be realized in this heterostructure multiferroics, leading to a design of spin field-effect transistor ${ }^{27,28}$.

The induced spin moment of surface Se is attributed to the exchange coupling $J \sim t^{2} / U$ between Te $p$ and Se $p$ orbitals, with $t$ the hopping constant and $U$ the intra-orbital Coulomb repulsion. In the limit of zero $t$ or infinite $U$, the system favors the triplet 


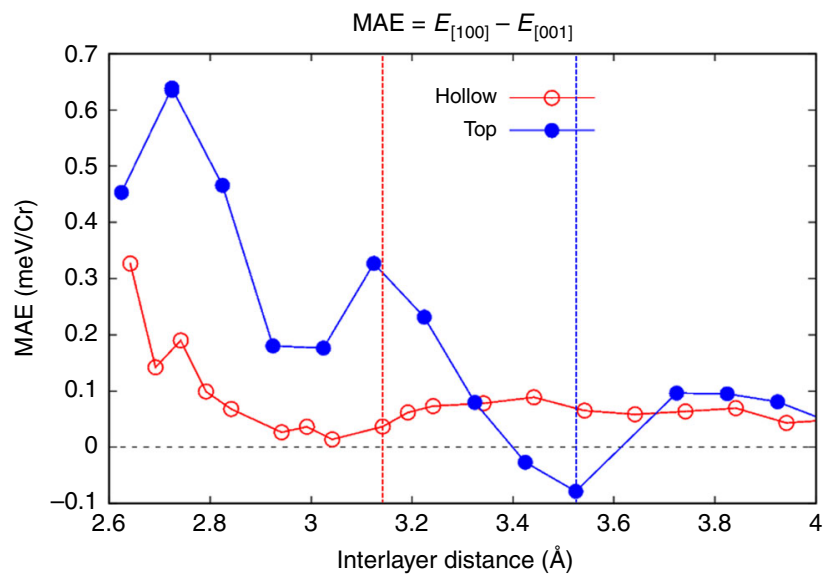

Fig. 3 Calculated magnetocrystalline anisotropy of $\mathrm{Cr}_{2} \mathrm{Ge}_{2} \mathrm{Te}_{6}$ in the heterostructure versus the vdW interlayer distance. Magnetocrystalline anisotropy energy (MAE) (in meV/Cr) of $\mathrm{Cr}_{2} \mathrm{Ge}_{2} \mathrm{Te}_{6}$ as a function of the distance to $\ln _{2} \mathrm{Se}_{3}$ in the $\mathbf{P}_{\mathbf{d n}}$ state, showing a gradual increase towards the positive MAE (easy-axis $\mathbf{S}_{\mathbf{C r}}$ ) upon decreasing the distance for both hollow and top stacking configurations. This set of calculations confirmed the scenario that the enhanced interfacial hybridization between $\mathrm{Cr}_{2} \mathrm{Ge}_{2} \mathrm{Te}_{6}$ and $\mathrm{In}_{2} \mathrm{Se}_{3}$ tends to switch the magnetocrystalline anisotropy from easy-plane to easy-axis. The calculation error is reflected by the symbol size $(\sim 0.01 \mathrm{meV} /$ $\mathrm{Cr}$ ). The red and blue dashed lines correspond to the equilibrium interfacial distances for hollow and top stacking configurations, respectively

state similar to the atomic Hund coupling, which is the case for Te $p$ and Se $p$. For a given value of $U$, the $t$ varies exponentially with the distance. Consistently, our calculations show the exponentially increasing Se spin moment with decreased interfacial distance, as shown in Fig. 4b. The correlation effect should depend on the specific nonlocal correlation functional. For different vdW functionals, the induced spin moments remain at nearly the same magnitude (Supplementary Fig. 6).

Discussion on practical experimental factors. It is of experimental guidance to remark on the possible effects of real material environments. Calculation and analysis in this work are based on the heterostructure of a bilayer system floating in vacuum. In the experimental realization, the initial anisotropy of the magnetic layer $\mathrm{Cr}_{2} \mathrm{Ge}_{2} \mathrm{Te}_{6}$ could be affected by a few factors, including contacting the materials of large dielectric constants ${ }^{22,29}$ or large SOC strength ${ }^{30}$, unintentional doping ${ }^{31-33}$ caused by chemicals in device fabrication process, and small amount of artificial strain induced in heterostructure preparation. These factors may affect the resultant magnetoelectric effect quantitatively, as reflected from the calculated MAE of the heterostructures based on the arbitrary sets of $U$ and $J$ values (see Supplementary Fig. 7): the increased $U$ value enhances the out-of-plane anisotropy, while the increased $J$ value enhances the in-plane anisotropy; for any tested set of $U$ and $J$ values, the out-of-plane anisotropy is always enhanced by $\sim 0.15 \mathrm{meV} / \mathrm{Cr}$ when the $\mathrm{In}_{2} \mathrm{Se}_{3}$ dipole is inverted from up to down. Therefore, even if our adopted values of $U$ and $J$ $(U=0.5 \mathrm{eV}, J=0.0 \mathrm{eV})$ slightly deviate from the exact description of the real heterostructure samples because of the aforementioned complex experimental conditions, the reversal of the $\mathrm{In}_{2} \mathrm{Se}_{3}$ polarization from up to down always strengthens the $2 \mathrm{D}$ ferromagnetic order in $\mathrm{Cr}_{2} \mathrm{Ge}_{2} \mathrm{Te}_{6}$. This leads to a general implication: in practice, one can always set a temperature so that 2D ferromagnetism could be found in $\mathrm{P}_{\mathrm{dn}}-\mathrm{In}_{2} \mathrm{Se}_{3}-\mathrm{Cr}_{2} \mathrm{Ge}_{2} \mathrm{Te}_{6}$ but disappear from $\mathrm{P}_{\mathrm{up}}-\mathrm{In}_{2} \mathrm{Se}_{3}-\mathrm{Cr}_{2} \mathrm{Ge}_{2} \mathrm{Te}_{6}$, leading to the practical switching experiments at finite temperatures. Therefore, the
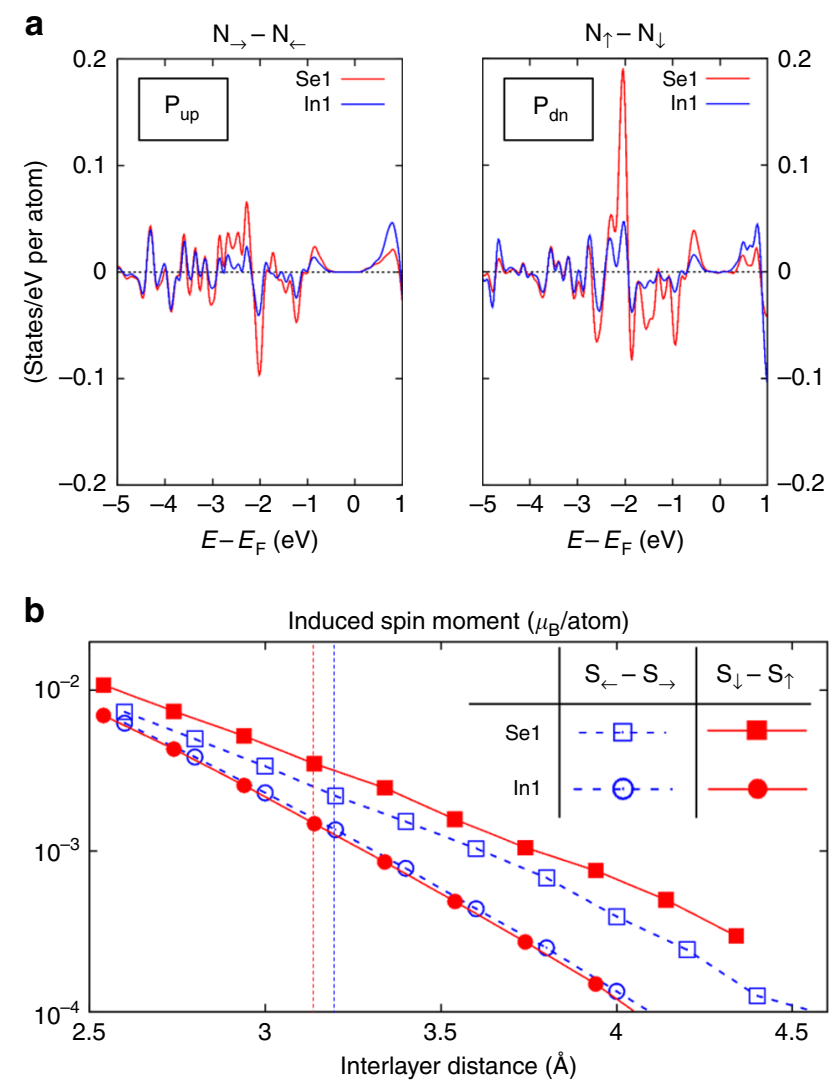

Fig. 4 Magnetoelectric effect in $\ln _{2} \mathrm{Se}_{3}$, mediated by the magnetic proximity to $\mathrm{Cr}_{2} \mathrm{Ge}_{2} \mathrm{Te}_{6}$. a Projected spin density of states for the surface (Se1) and subsurface ( $\ln 1)$ atomic layers close to $\mathrm{Cr}_{2} \mathrm{Ge}_{2} \mathrm{Te}_{6}$. Left and right panels correspond to the heterostructures with $\mathbf{P}_{\mathbf{u p}}$ and $\mathbf{P}_{\mathbf{d n}} \ln _{2} \mathrm{Se}_{3}$, respectively, with the in-plane and out-of-plane spin quantization axis. Given the prohibited long-range magnetic order for the easy-plane 2D magnetic system, the spin-polarized $\ln _{2} \mathrm{Se}_{3}$ as shown by the left panel of (a) is only a ground state at zero Kelvin. But the easy-axis spin-polarized $\ln _{2} \mathrm{Se}_{3}$ as shown by the right panel of (a) is practical at finite temperatures. Hence, the magnetization of the semiconducting $\ln _{2} \mathrm{Se}_{3}$ can be switched by its own electric polarization, while in proximity to $\mathrm{Cr}_{2} \mathrm{Ge}_{2} \mathrm{Te}_{6}$. b Interlayer distance dependence of the proximity-induced Se1 and In1 spin moments. The vertical dashed lines denote the equilibrium distances predicted by PBE with D2 vdW correction. Note that the induced spin moments are negative, which means the interlayer antiferromagnetic proximity effect in the studied $\mathrm{In}_{2} \mathrm{Se}_{3}-\mathrm{Cr}_{2} \mathrm{Ge}_{2} \mathrm{Te}_{6}$ heterostructures

magnetoelectric effect presented here, based on the modification of MAE by the intricate interface hybridization which further relates to the electric polarization of the $2 \mathrm{D}$ ferroelectrics, is an intrinsic interfacial phenomenon.

Summary. We employed first-principles DFT calculations on a vdW heterostructure consisting of ferromagnetic $\mathrm{Cr}_{2} \mathrm{Ge}_{2} \mathrm{Te}_{6}$ and ferroelectric $\mathrm{In}_{2} \mathrm{Se}_{3}$ monolayers. By reversing the electric polarization of $\mathrm{In}_{2} \mathrm{Se}_{3}$, the calculated magnetocrystalline anisotropy of $\mathrm{Cr}_{2} \mathrm{Ge}_{2} \mathrm{Te}_{6}$ changes between easy-axis and easy-plane (i.e., switching on/off of the ferromagnetic order), which promises a novel design of magnetic memory. Furthermore, $\mathrm{In}_{2} \mathrm{Se}_{3}$ becomes magnetic ferroelectrics, with switchable spin polarizations according to its own electric polarization. The $2 \mathrm{D}$ multiferroic heterostructures would tremendously enlarge the landscape of multiferroics by artificially assembling 2D layers and provide new material platforms for a plethora of emergent interfacial phenomena. 


\section{Methods}

The DFT method and parameters. All the calculations were performed by the DFT method implemented in Vienna ab initio Simulation Package (VASP) ${ }^{34}$, with the Perdew-Burke-Ernzerhof (PBE) functional ${ }^{35}$ in the scheme of generalized gradient approximation (GGA). The main data was calculated by GGA + U based on the Liechtenstein approach with $U=0.5 \mathrm{eV}$ and $J=0.0 \mathrm{eV}$. The van der Waals interatomic forces are described by the D2 Grimme method ${ }^{36}$. The K-mesh of $6 \times$ $6 \times 1$ and the energy cutoff of $300 \mathrm{eV}$ are used for the structural optimization. The dipole correction is included to exclude spurious dipole-dipole interaction between periodic images.

\section{Data availability}

The data that support the findings of this study are available from the corresponding author upon reasonable request.

Received: 22 September 2018 Accepted: 16 May 2019

Published online: 14 June 2019

\section{References}

1. Wang, J. et al. Epitaxial $\mathrm{BiFeO}_{3}$ multiferroic thin film heterostructures. Science 299, 1719 (2003).

2. Kimura, T. et al. Magnetic control of ferroelectric polarization. Nature 426, 55 (2003).

3. Ramesh, R. \& Spaldin, N. A. Multiferroics: progress and prospects in thin films. Nat. Mater. 6, 21 (2007).

4. Fiebig, M. et al. Revival of the magnetoelectric effect. J. Phys. D. 38, R123 (2005).

5. Fiebig, M. et al. The evolution of multiferroics. Nat. Rev. Mater. 1, 16046 (2016).

6. Cheong, S.-W. \& Mostovoy, M. Multiferroics: a magnetic twist for ferroelectricity. Nat. Mater. 6, 13 (2007).

7. Hill, N. A. Why are there so few magnetic ferroelectrics? J. Phys. Chem. B 104, 6694 (2000)

8. Zheng, H. et al. Multiferroic $\mathrm{BaTiO}_{3}-\mathrm{CoFe}_{2} \mathrm{O}_{4}$ nanostructures. Science 303, 661 (2004).

9. Geim, A. K. \& Grigorieva, I. V. Van der Waals heterostructures. Nature 499, 419 (2013).

10. Gong, C. et al. Discovery of intrinsic ferromagnetism in two-dimensional van der Waals crystals. Nature 546, 265-269 (2017).

11. Huang, B. et al. Layer-dependent ferromagnetism in a van der Waals crystal down to the monolayer limit. Nature 546, 270-273 (2017).

12. Gong, C. \& Zhang, X. Two-dimensional magnetic crystals and emergent heterostructure devices. Science 363, eaav4450 (2019).

13. Liu, F. et al. Room-temperature ferroelectricity in $\mathrm{CuInP}_{2} \mathrm{~S}_{6}$ ultrathin flakes Nat. Commun. 7, 12357 (2016).

14. Seixas, L., Rodin, A. S., Carvalho, A. \& Castro Neto, A. H. Multiferroic twodimensional materials. Phys. Rev. Lett. 116, 206803 (2016).

15. Wang, H. \& Qian, X. Two-dimensional multiferroics in monolayer group IV monochalcogenides. 2D Mater. 4, 015042 (2017)

16. Luo, W., Xu, K. \& Xiang, H. Two-dimensional hyperferroelectric metals: a different route to ferromagnetic-ferroelectric multiferroics. Phys. Rev. B 96, 235415 (2017).

17. Qi, J., Wang, H., Chen, X. \& Qian, X. Two-dimensional multiferroic semiconductors with coexisting ferroelectricity and ferromagnetism. Appl. Phys. Lett. 113, 043102 (2018).

18. Ding, W. et al. Prediction of intrinsic two-dimensional ferroelectrics in $\mathrm{In}_{2} \mathrm{Se}_{3}$ and other $\mathrm{III}_{2}-\mathrm{VI}_{3}$ van der Waals materials. Nat. Commun. 8, 14956 (2017).

19. Zhou, Y. et al. Out-of-plane piezoelectricity and ferroelectricity in a-layered $\mathrm{In}_{2} \mathrm{Se}_{3}$ nanoflakes. Nano Lett. 17, 5508-5513 (2017).

20. Cui, C. et al. Intercorrelated in-plane and out-of-plane ferroelectricity in ultrathin two-dimensional layered semiconductor $\operatorname{In}_{2} \mathrm{Se}_{3}$. Nano Lett. 18, 1253-1258 (2018).

21. Xiao, J. et al. Intrinsic two-dimensional ferroelectricity with dipole locking. Phys. Rev. Lett. 120, 227601 (2018)

22. Mermin, N. D. \& Wagner, H. Absence of ferromagnetism or antiferromagnetism in one- or two-dimensional isotropic Heisenberg models. Phys. Rev. Lett. 17, 1133 (1966).

23. Bruno, P. Magnetization and Curie temperature of ferromagnetic ultrathin films: the influence of magnetic anisotropy and dipolar interactions. Mater. Res. Symp. Proc. 231, 299-310 (1991).

24. Henkelman, G. A climbing image nudged elastic band method for finding saddle points and minimum energy paths. J. Chem. Phys. 113, 9901-9904 (2000).

25. Wang, D. et al. First-principles theory of surface magnetocrystalline anisotropy and the diatomic-pair model. Phys. Rev. B 47, 14932 (1993).
26. Sui, X. et al. Voltage-controllable colossal magnetocrystalline anisotropy in single-layer transition metal dichalcogenides. Phys. Rev. B 96, 041410 (2017).

27. Datta, S. \& Das, B. Electronic analog of the electro-optic modulator. Appl. Phys. Lett. 56, 665-667 (1990).

28. Gong, S.-J. et al. Electrically induced $2 \mathrm{D}$ half-metallic antiferromagnets and spin field effect transistors. Proc. Natl Acad. Sci. USA 115, 8511-8516 (2018).

29. Jiang, S., Shan, J. \& Mak, K. F. Electric-field switching of two-dimensional van der Waals magnets. Nat. Mater. 17, 406-410 (2018).

30. Avsar, A. et al. Spin-orbit proximity effect in graphene. Nat. Commun. 5, 4875 (2014).

31. Huang, B. et al. Electrical control of $2 \mathrm{D}$ magnetism in bilayer $\mathrm{CrI}_{3} . \mathrm{Nat}$. Nanotechnol. 13, 544-548 (2018).

32. Jiang, S., Li, L., Wang, Z., Mak, K. F. \& Shan, J. Controlling magnetism in 2D $\mathrm{CrI}_{3}$ by electrostatic doping. Nat. Nanotechnol. 13, 549-553 (2018).

33. Wang, Z. et al. Electric-field control of magnetism in a few-layered van der Waals ferromagnetic semiconductor. Nat. Nanotechnol. 13, 554-559 (2018).

34. Kresse, G. \& Furthmuller, J. Efficiency of ab-initio total energy calculations for metals and semiconductors using a plane-wave basis set. Comput. Mater. Sci. 6, 15-50 (1996)

35. Perdew, J. P., Burke, K. \& Ernzerhof, M. Generalized gradient approximation made simple. Phys. Rev. Lett. 77, 3865 (1996).

36. Grimme, S. Semiempirical GGA-type density functional constructed with a long-range dispersion correction. J. Comp. Chem. 27, 1787 (2006).

\section{Acknowledgements}

C.G., Y.W., and X.Z. acknowledge the support from the U.S. Department of Energy, Office of Science, Basic Energy Sciences, Materials Sciences and Engineering Division under contract no. DE-AC02-05-CH11231 within the van der Waals Heterostructures program (KCWF16) for the conceptual development and preliminary calculations of 2D heterostructure multiferroics. The support from the National Science Foundation (NSF) under Grant 1753380 for the calculation and analysis of 2D magnets and the King Abdulah University of Science and Technology (KAUST) Office of Sponsored Research (OSR) under Award OSR-2016-CRG5- 2996 for the calculation and analysis of 2D ferroelectrics was also acknowledged. G.L. acknowledges the support by the National Research Foundation of Korea (Basic Science Research Program: 2018R1D1A1B07045983) for the systematic computational studies of 2D heterostructure multiferroics. Computation was supported by KISTI (KSC-2018-CRE-0048).

\section{Author contributions}

C.G. and X.Z. conceived the project. G.L. and E.M.K. performed the calculations with the close discussions with C.G. C.G., G.L., and X.Z. did data analysis and wrote the paper with the assistance from Y.W.

\section{Additional information}

Supplementary Information accompanies this paper at https://doi.org/10.1038/s41467 019-10693-0.

Competing interests: The authors declare no competing interests.

Reprints and permission information is available online at http://npg.nature.com/ reprintsandpermissions/

Peer review information: Nature Communications thanks the anonymous reviewers for their contribution to the peer review of this work

Publisher's note: Springer Nature remains neutral with regard to jurisdictional claims in published maps and institutional affiliations.

Open Access This article is licensed under a Creative Commons Attribution 4.0 International License, which permits use, sharing, adaptation, distribution and reproduction in any medium or format, as long as you give appropriate credit to the original author(s) and the source, provide a link to the Creative Commons license, and indicate if changes were made. The images or other third party material in this article are included in the article's Creative Commons license, unless indicated otherwise in a credit line to the material. If material is not included in the article's Creative Commons license and your intended use is not permitted by statutory regulation or exceeds the permitted use, you will need to obtain permission directly from the copyright holder. To view a copy of this license, visit http://creativecommons.org/ licenses/by/4.0/

(C) The Author(s) 2019 